\title{
Design of High Speed Permanent Magnet Synchronous Linear Motor Control System
}

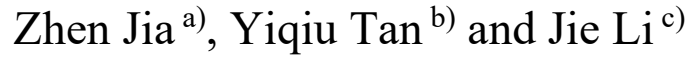 \\ Engineering Research Center of Maglev Technology, National University of Defense Technology, Changsha, \\ 410073, China. \\ a)1031917012@qq.com, b) 522346716@qq.com, ${ }^{c}$ jieli@nudt.edu.cn
}

\begin{abstract}
The propulsion technology of high speed linear motor is a hot spot in the field of motor field in recent years. This technology has a wide application prospect. In this paper, based on the working principle of permanent magnet synchronous linear motor, a driver of permanent magnet synchronous linear motor is designed.
\end{abstract}

Key words: High speed permanent magnet synchronous motor(PMSM), motor driver, communication monitoring system.

\section{INTRODUCTION}

High speed linear motor has always been the high-tech research of all countries. High speed linear motor plays a key role in electromagnetic launch, aerospace booster and other fields. Permanent magnet synchronous linear motor (PMSM) is the good choice for high-speed propulsion because of its large thrust, high speed, high accuracy, fast response and high positioning accuracy $[1,2]$.

The key to ensure the normal operation of motor is the motor driver. How to design a reasonable and reliable motor driver directly affects the performance of the motor. In this paper, a permanent magnet synchronous linear motor driver with good performance and strong reliability is designed for the real permanent magnet synchronous linear motor.

\section{THE WORKING PRINCIPLE OF THE MOTOR}

The linear synchronous motor is evolved from the corresponding rotating motor, and its working principle is similar to the ordinary rotating motor. Compared with linear induction motor, linear synchronous motor has greater driving force and better control performance and position precision. Therefore, the application of linear synchronous motor in the drive system of high speed ground transportation and straight-line lifting device is more and more important. All kinds of linear synchronous motors are the main choice of linear driving [3].

The magnetic poles of a linear synchronous motor can be excited by a DC excitation winding or by a permanent magnet, in which the reliability and efficiency of the permanent magnet are higher. The working principle of the permanent magnet linear synchronous motor is shown in Figure 1, 1 in the diagram is the traveling wave magnetic field, 2 is the secondary, and the 3 is the primary. 


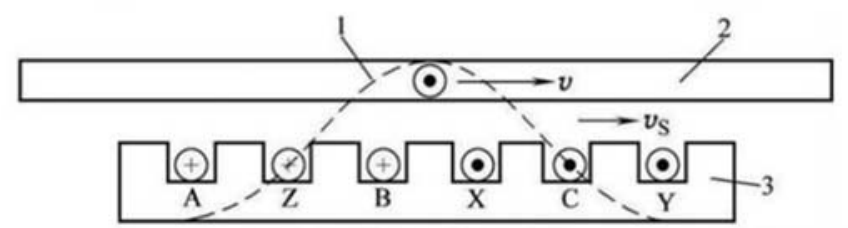

FIG. 1. The working principle of permanent magnet synchronous linear motor

The three-phase sinusoidal AC current is entered into the three-phase stator winding, and then under the common effect of air gap travelling magnetic field and magnetic pole magnetic field of permanent magnet, the air gap magnetic field produces the electromagnetic thrust on the permanent magnetic pole. If the primary is fixed, the magnetic pole moves in a straight line along the direction of the traveling wave magnetic field under the thrust of the electromagnetic, and the velocity of the magnetic pole is the same as that of the traveling wave field [4,5]. Based on the hardware structure of the permanent magnet synchronous linear motor, the control system of the motor is designed. The motor structure is shown in Figure 2.

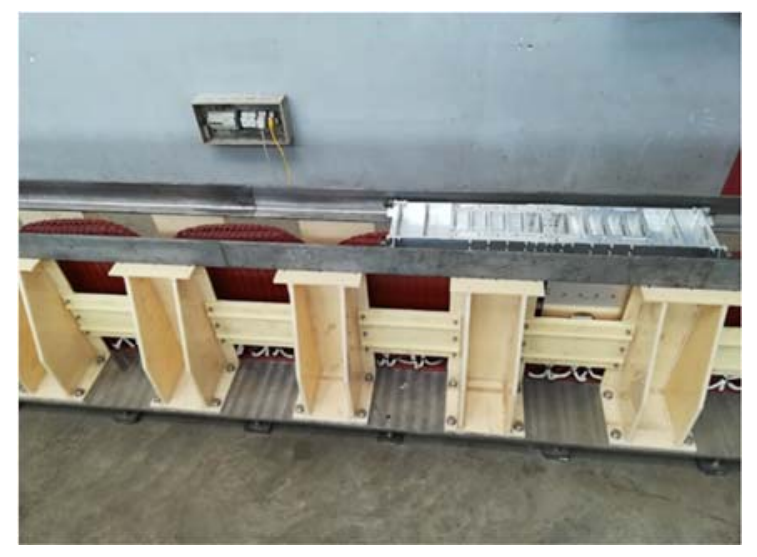

FIG. 2. Permanent magnet synchronous linear motor

\section{DESIGN OF CONTROL SYSTEM}

The control system of permanent magnet synchronous linear motor can be divided into strong electric circuit, weak electric circuit and communication monitoring system. The strong electric circuit provides the power supply for the motor, which is used to produce three alternating current. The weak electric circuit controls the motor in real time according to the motor state of the motor. The communication monitoring system achieves the real-time communication between the PC and the control board, and achieves the monitoring, recording and real-time control of the motor running state.

\section{The Design of the Strong Electric Circuit}

The strong electric circuit is mainly composed of DC power input, charge resistance, main contractor, charge capacitor, three full bridge chopper circuit, and reactor. The DC rated input voltage of the strong electric circuit of the motor used for driving the hardware is 330V.The pre-charge switch KM1 is closed. The current is limited by the power resistor R1 and R2, and then the capacitor is charged. After the capacitor is charged, the main contact switch KM2 is closed. The full bridge chopper circuit can control the output current of three motors by the output of PWM which is controlled by the control board, so as to drive the motor to run at high speed. 


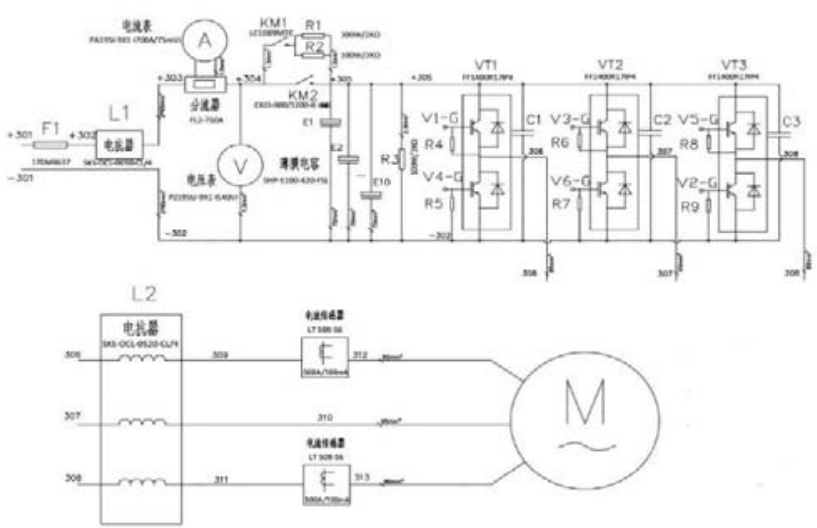

FIG. 3. The strong electric circuit diagram of the motor

\section{The Design of Weak Electric Circuit}

The working principle of weak current circuit for high speed permanent magnet linear synchronous motor is shown in figure 4. Three current sensors are selected to measure DC input total current and two-phase currents. Three channel voltage sensors are selected to measure DC input voltage and two phase-phase voltages. A high-precision wire drawing sensor is selected to measure motor position and acceleration sensor to measure motor thrust. There are eight sensor signals. The core control chip selects the classic motor control chip 28335DSP. The AD sampling module selects 16bit AD7606 chip with high accuracy. The FPGA chip is added to the control circuit to increase the number of IO ports and enhance the data computing ability of the system. The signal of the eight-channel sensor is filtered and amplified by analog circuit. After sampling by AD7606 chip, it is transmitted to the core control panel 28335DSP through FPGA chip. The core control algorithm outputs the PWM wave after calculation, so as to drive the strong power full bridge chopper circuit to control the output of three-phase circuit and achieve real-time control of motor.

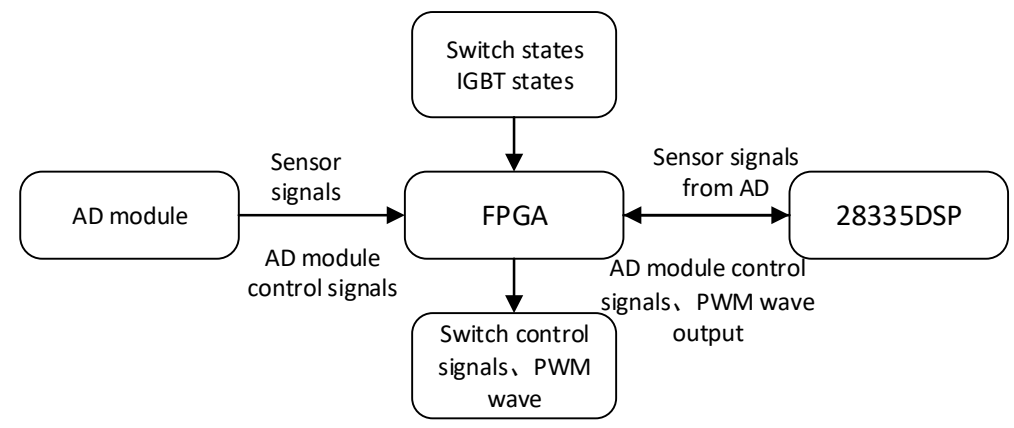

FIG. 4. The working principle of the control board

FPGA, that is field programmable gate array. Thanks to its rich IO resources and programmable logic timing function, it solves the problem of insufficient number of IO ports in 28335DSP control panel in weak current control circuit. At the same time, the logic timing control of the system is enhanced. In this system, FPGA has 12 IO ports for output and 12 ports for input. Its operation is shown in Table 1. Before the motor drive begins to work, the DSP control the FPGA output port to turn on the KM1 switch to charge the capacitor. After the charge is completed, the strong electric circuit is turned on through the KM2. At the same time, the external running indicator lights up. If there is a error, the corresponding error indicator lights up, indicating the system fault. During the operation of the system, the input port reads the hardware status of the system to monitor the running state and ensure that the strong part of the system works normally. 
TABLE 1. IO usage

\begin{tabular}{cccc}
\hline Output & Function & Input & Function \\
\hline Q0 & KM1 switch & I0 & KM1 state feedback \\
Q1 & KM2 switch & I1 & KM2state feedback \\
Q2 & Running light switch & I2 & Stop button feedback \\
Q3 & IGBT error & I3 & KM3 state feedback \\
Q4 & KM3 switch & I4 & IGBT high temperature alarm1 \\
Q5-11(I6-11) & spare & I5 & IGBT high temperature alarm 2 \\
\hline
\end{tabular}

\section{Communication and Monitoring System}

Communication monitoring system is based on data communication between upper monitor and control board. The real-time monitoring and control of motor is achieved by monitoring and recording interface on upper monitor. The communication module, using the ENC28J60QFN network communication chip as the intermediary, achieves the transformation of the SPI port of the DSP into the network port. And the UDP communication protocol is used to achieve the data communication between the control board and the PC. The host computer's control signals and the drive state of the slave control board and the operation data of the motor interact with each other through compression and decompression. The circuit diagram of the converter of SPI port to the network port is shown in Figure 5.

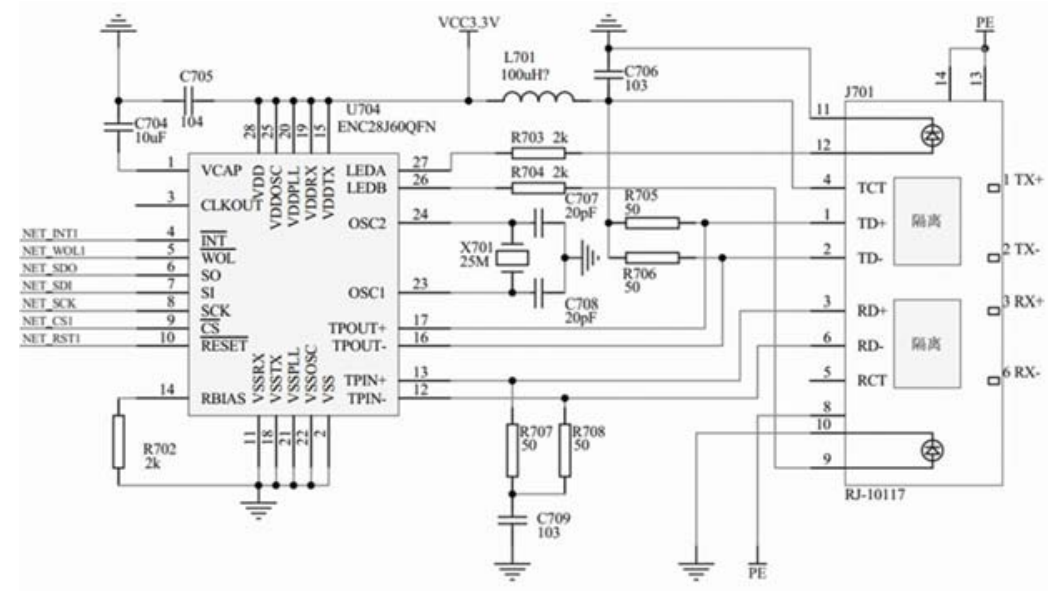

FIG. 5. The circuit diagram of the converter of SPI port to the network port

The interface between host computer and motor is designed as shown in Figure 6. The interface is written by $\mathrm{C}$ language program. By setting the fixed IP, the communication between host computer and slave computer is achieved. As you can see from the monitoring interface, the interface's functions include setting up the expected phase current value, the current loop ratio coefficient, the speed limit and the distance limitation during the operation of the motor. Moreover, the control of the opening and closing of PWM waves and controlling the motor automatically reset to the initial location by back $=3$ button is included. At the same time, during the running of motor, the interfere can show the location of motor running in real time (displacement $\mathrm{P} 1$ ), A phase current, $\mathrm{C}$ phase current, input total voltage, $\mathrm{AB}$ phase voltage and motor fault signal and other relevant data, so as to achieve real-time monitoring and control. 


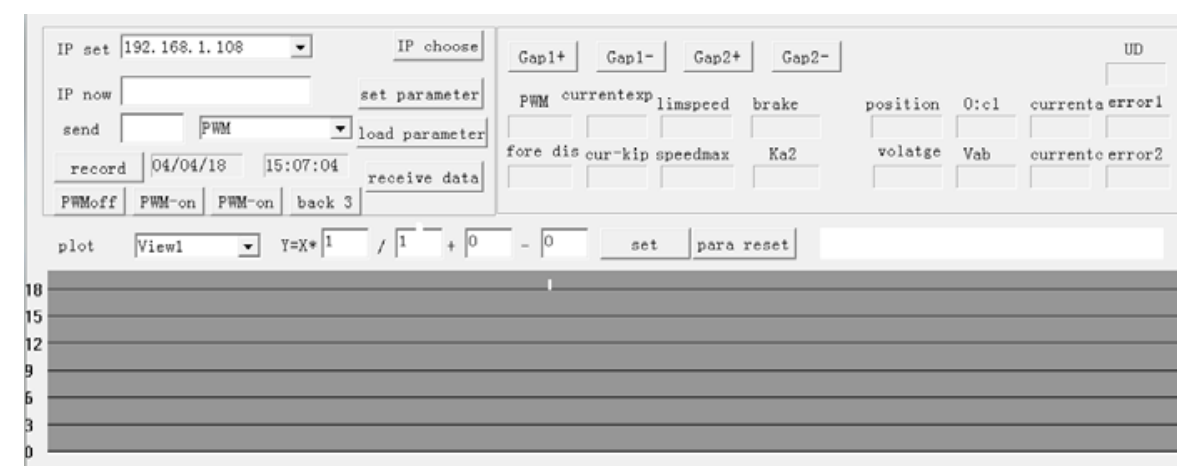

FIG. 6. Motor monitoring interface

\section{CONCLUSION}

According to the performance characteristics of motor, we must design a reasonable motor driver to give full play to the motor performance. In this paper, based on the working principle of permanent magnet synchronous motor, according to the characteristics of the permanent magnet synchronous linear motor, the design of the strong electric circuit, the design of the weak electric circuit and the communication monitoring system are introduced in detail, and the complete motor driver is designed. The motor drive system has been tested in practice, and its performance is running well, with complete functions, practicability and reliability.

\section{ACKNOWLEDGEMENTS}

This work was supported by the National Key R\&D Program of China [grant number 2016YFB1200601-B12]; the research Plan Program of National University of Defense Technology [grant number 2K16-02-02]; the Opening Foundation of the State Key Laboratory of Functional Materials for Informatics [grant number SKL-2017-07].

\section{REFERENCES}

1. Kun Tao, Chunmei Wang, Liqiong Zhang. A review of permanent magnet synchronous linear motor control system [J]. Coal Mine Machinery, 2014, (5): 14-16.

2. Jizhu Liu. Research on permanent magnet linear synchronous motor drive system for electromagnetic catapult[D]. Harbin Institute of Technology, 2010.

3. Rui Peng. Research on driving and control strategy of permanent magnet synchronous linear motor[D]. Guangdong University of Technology, 2015. DOI:10.7666/d. Y2795783.

4. Minghui Dang. Research on Permanent Magnet Synchronous Linear Motor Control System Based on DSP [D]. Zhejiang Sci-Tech University, 2015.

5. Chuansheng Tang. Research on control method of permanent magnet synchronous linear motor[D]. University of Electronic Science and Technology of China, 2014. 